\title{
Velocity Selection Problem in the Presence of the Triple Junction
}

\author{
E. A. Brener, C. Hüter, D. Pilipenko, and D. E. Temkin \\ Institut für Festkörperforschung, Forschungszentrum Jülich, D-52425 Jülich, Germany
}

(Received 16 April 2007; published 7 September 2007)

\begin{abstract}
Melting of a bicrystal along the grain boundary is discussed. A triple junction plays a crucial role in the velocity selection problem in this case. In some range of the parameters an entirely analytical solution of this problem is given. This allows us to present a transparent picture of the structure of the selection theory. We also discuss the selection problem in the case of the growth of a "eutectoid dendrite."
\end{abstract}

PACS numbers: 64.70.Dv, 45.70.Qj, 68.08.-p, 81.30.Fb

During the last decades, our understanding of pattern formation in various nonlinear dissipative systems has made remarkable progress. Building on this foundation, it has now become possible to develop a description of a large class of patterns that are found in diffusional growth. In this classical problem of dendritic growth, velocity selection is controlled by tiny singular effects of the anisotropy of the surface energy (see, for example, $[1,2]$ ). In the case of isotropic surface energy, the dendritic solution does not exist and instead the so-called doublon structure is the solution of the problem $[3,4]$. The dendriticlike structures which occur during melting have also been observed experimentally (see [5] and references therein). Moreover, for many applications the investigation of the influence of the defects on the melt-crystal interface behavior is of great importance.

In this Letter we discuss the melting process along the grain boundary in the crystal (see Fig. 1). The presence of a triple junction at the tip of the melting zone leads to an entirely different selection mechanism, since the triple junction produces a very strong perturbation of the liquid-solid interfaces and weak anisotropy effects can be neglected. This idea was expressed in [6]. We also discuss the importance of a triple junction for the selection problem of the growth of a "eutectoid dendrite" (see Fig. 2).

Melting along a grain boundary. - We consider the two dimensional melting problem of a pure bicrystal with a straight grain boundary as shown in Fig. 1. Overheating of the crystal leads to a moving melt zone. The contact angle $\phi$ at the triple junction is related to the surface energies by Young's law: $2 \gamma \cos \phi=\gamma_{b}$, where $\gamma$ is the surface energy on the liquid-solid interface and $\gamma_{b}$ is the surface energy of the grain boundary. We introduce the dimensionless temperature field $u=c_{p}\left(T_{\infty}-T\right) / L$, where $L$ is the latent heat, $c_{p}$ is heat capacity, and $T_{\infty}$ is the temperature in the crystal far away from the interface.

The temperature field $u$ obeys the following heat diffusion equation and boundary conditions

$$
\begin{gathered}
D \nabla^{2} u=\partial u / \partial t, \\
v_{n}=D \vec{n}\left(\left.\vec{\nabla} u_{L}\right|_{\text {int }}-\left.\vec{\nabla} u_{S}\right|_{\text {int }}\right),
\end{gathered}
$$

$$
\Delta-d_{0} \kappa=\left.u\right|_{\text {int }} \text {, }
$$

where $L$ and $S$ refer to the liquid and solid, respectively, $d_{0}=\gamma T_{M} c_{p} / L^{2}$ is the capillarity length, $T_{M}$ is the melting temperature, and $\kappa$ is the curvature of the liquid-solid interface, which assumed to be positive for convex interfaces (as in Fig. 1). We introduce the dimensionless overheating $\Delta=c_{p}\left(T_{\infty}-T_{M}\right) / L$, and the Peclet number $p=v R / 2 D$ with $D$ being the thermal diffusion constant (assumed to be the same in both phases), $R$ is the radius of curvature of the asymptotically fitted Ivantsov parabola and $v$ is the velocity of the steady-state motion.

The physics underlying Eqs. (1)-(3) is quite simple. A moving melting front absorbs the latent heat. Requirements of the heat conservation at the interface give us Eq. (2) (n is the normal to the front pointing into the solid phase; $v_{n}$ is the normal velocity of the front). The local thermodynamical equilibrium in the interfacial region implies Eq. (3). This is the Gibbs-Tomson relation, which gives the equilibrium value of the temperature at the interface taking into account curvature corrections.

An equivalent formulation of the problem which is more convenient for our purposes is obtained by eliminating the thermal field. This can be done by using the standard Green's function techniques to obtain an equation in closed

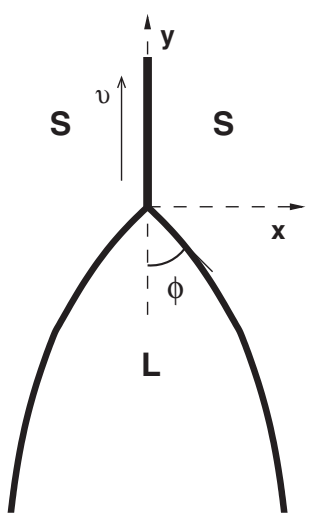

FIG. 1. Propagation of the melt zone along the grain boundary. $S$ corresponds to two solid grains and $L$ corresponds to the liquid phase. The triple junction is at the origin of the coordinate system. 


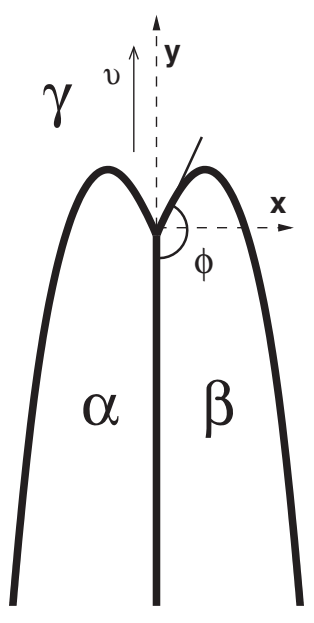

FIG. 2. Schematic picture of the interface structure in the eutectoid reaction.

form for the shape of the solid-liquid interface (see, for example [7]). In the comoving frame of reference the steady-state equation reads

$$
\Delta(p)-\frac{d_{0}}{R} \kappa=\frac{p}{\pi} \int_{-\infty}^{\infty} d x^{\prime} e^{-p\left(y-y^{\prime}\right)} K_{0}\left(p \eta\left(x, x^{\prime}\right)\right),
$$

where $\eta=\left[\left(x-x^{\prime}\right)^{2}+\left(y-y^{\prime}\right)^{2}\right]^{1 / 2}$, and $K_{0}$ is the modified Bessel function of third kind in zeroth order; all lengths are measured in units of $R$. The relation between the overheating $\Delta$ and the Peclet number $p$ is given by the Ivantsov relation [8]: $\Delta=\sqrt{p} \bar{\pi} \exp (p) \operatorname{erfc}(\sqrt{p})$.

Equation (4) is a complicated nonlinear integrodifferential equation. We should find a smooth solution of this equation which has a proper angle at the tip and which is close to the Ivantsov parabola $\left(y=-x^{2} / 2\right)$ in the tail region. The classical dendritic growth problem does not have a solution with isotropic surface tension and with a smooth tip which corresponds to the angle $\phi=\pi / 2$ $[1,2]$. This statement can be expressed in the following form. For any given positive values of the Peclet number $p$ and the so-called stability parameter $\sigma=d_{0} / p R$ the symmetric solution which is close to the Ivantsov parabola in the tail region has an angle at the tip $\phi=f(\sigma, p)<\pi / 2$. The limit $\sigma=0$ and $\phi=\pi / 2$ is a singular limit for that problem. For example, Meiron [9] numerically calculated the angle $\phi$ as a function of $\sigma$ for several values of the Peclet number with isotropic surface tension and found that the angle $\phi$ remains smaller than $\pi / 2$ for any positive $\sigma$.

Our problem differs from the classical dendritic problem precisely in that we need to satisfy the condition that the angle $\phi$ at the tip should be smaller than $\pi / 2$. This means that we can select the stability parameter $\sigma=\sigma^{\star}(\phi, p)$ as a function of $\phi$ and $p$. In order to illuminate this selection mechanism we consider the case of small opening angles, i.e., $\phi \ll 1$ and small overheating $(p \ll 1)$ which allows us to drastically simplify the problem and finally obtain the selection in some limit entirely analytically.

For small opening angles $\phi$, the following rescaling of coordinates is useful in order to eliminate small parameters from the interface shape: $x \rightarrow x / \phi, y \rightarrow y / \phi^{2}$. After this rescaling, $y=-|x|$ near the tip and in the tail region the asymptotic of the Ivantsov parabola $\left(y=-x^{2} / 2\right)$ remains unchanged. In this small angle approximation the function $\eta$ depends only on the $y$ variable, $\eta \approx\left|y-y^{\prime}\right| \phi^{-2}$, because $x \ll y$. Consequently the whole integral kernel in Eq. (4), under the assumption of small Peclet numbers, is a function only of $y$. Because of the symmetry of the interface we consider it only for $x>0$. It is more convenient to treat the shape as a function $x(y)$ and to make a change of variables, $y \rightarrow-y$. The curvature of the interface can be written as $\kappa \approx-d^{2} x / d y^{2}$. Performing all these steps we reduce the original nonlinear Eq. (4) to the following linear equation:

$$
\begin{aligned}
1+\mu_{d} \frac{d^{2} x}{d y^{2}}= & \frac{2}{\pi} \sqrt{\frac{p}{\pi \phi^{2}}} \int_{0}^{\infty} d y^{\prime} \frac{d x\left(y^{\prime}\right)}{d y^{\prime}} \\
& \times e^{-\left(p / \phi^{2}\right)\left(y^{\prime}-y\right)} K_{0}\left(\frac{p}{\phi^{2}}\left|y^{\prime}-y\right|\right),
\end{aligned}
$$

where $\mu_{d}=d_{0} \phi^{3} / R \Delta=\sigma \phi^{3} \sqrt{p / \pi}$. As follows from Eq. (5), the eigenvalue $\mu_{d}$ is a function only of one parameter, $p / \phi^{2}$. We solve Eq. (5) numerically using standard linear algebra routines. The results are presented in Fig. 3.

For small values of $p / \phi^{2} \ll 1$, the eigenvalue $\mu_{d}$ is proportional to the prefactor in front of the integral term in Eq. (5), i.e., $\mu_{d}=\alpha \sqrt{\left(p / \phi^{2}\right)}$. We found numerically that $\alpha \approx 2$.2, which leads to the following scaling relations for

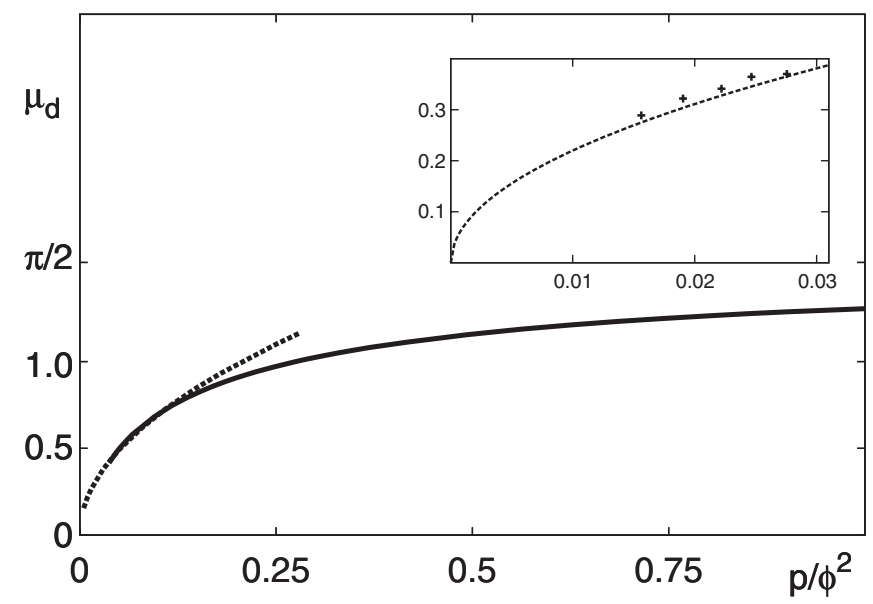

FIG. 3. Eigenvalue $\mu_{d}$ as a function of $p / \phi^{2}$. Solid line represents the numerical solution of Eq. (5), dashed line corresponds to the asymptotic behavior $\mu_{d}=2.2 \sqrt{p / \phi^{2}}$. The inset shows an enlarged region of small values of $p / \phi^{2}$, the dashed line is $2.2 \sqrt{p / \phi^{2}}$, crosses correspond to the data extracted from Ref. [9]. 
the selected tip radius and velocity: $R / d_{0} \approx 0.81 \phi^{4} \Delta^{-2}$ and $v d_{0} / D \approx 0.79 \phi^{-4} \Delta^{4}$. We compare our asymptotic predictions $\mu_{d}=2.2 \sqrt{p / \phi^{2}}$, with data extracted from Fig. (1c) of Ref. [9]. Despite the fact that the results of Ref. [9] were obtained by the solution of the full Eq. (4), and our results were obtained under the assumption of small opening angles, they show a relatively good quantitative agreement up to angles $\phi \approx \pi / 6$ (see the inset in Fig. 3).

In the case of large value of $p / \phi^{2} \gg 1$ further simplifications of Eq. (5) are possible. The combination of the exponential term with the asymptotic of the Bessel function for the large arguments leads to the integral kernel $1 / \sqrt{p \phi^{-2}\left(y-y^{\prime}\right)}$ for the argument $y^{\prime}<y$, while, for $y^{\prime}>$ $y$ the integral kernel is exponentially small and can be neglected. Thus, Eq. (5) finally reads

$$
1+\mu_{d} \frac{d^{2} x}{d y^{2}}=\frac{\sqrt{2}}{\pi} \int_{0}^{y} \frac{d x\left(y^{\prime}\right)}{d y^{\prime}} d y^{\prime} \frac{1}{\sqrt{y-y^{\prime}}} .
$$

This equation for the unknown function $f(y)=d x / d y$ can be solved by means of the Laplace transformation

$$
F(s)=\int_{0}^{\infty} f(y) \exp (-s y) d y .
$$

Using the boundary condition, $f(0)=1$, we get

$$
F(s)=\left(\mu_{d} \sqrt{s}-1 / \sqrt{s}\right)\left(\mu_{d} s^{3 / 2}-\sqrt{2 / \pi}\right)^{-1} .
$$

The function $F(s)$ has a pole at $s=\left(2 / \pi \mu_{d}^{2}\right)^{1 / 3}$. Therefore, the physically relevant solution exists only if the parameter $\mu_{d}=\pi / 2$, which means that the pole and zero of the function $F(s)$ coincide. To our best knowledge this is the first example where the problem of velocity selection in dendritic growth is solved entirely analytically. This exact analytical treatment demonstrates in a very transparent way why the physically relevant solution of Eq. (7) exists only for some specific value of the parameter $\mu_{d}$. The inverse Laplace-transform of Eq. (7) finally gives the closed expression for the derivative of the interface profile:

$$
\begin{aligned}
d x / d y= & {[(\sqrt{3}+i) / 2 \sqrt{3}] \exp [(-1+i \sqrt{3}) y / \pi] } \\
& \times \operatorname{erfc}[(1+i \sqrt{3}) \sqrt{y / 2 \pi}]+\text { c.c. }
\end{aligned}
$$

We note that the same analytical treatment is possible also in the case where interface kinetic effects are included. The interface kinetics requires an additional term, $-v_{n} / \beta$, in the left-hand side of Eq. (4) and, correspondingly, the term $-\mu_{\beta} d x / d y$ in Eqs. (5) and (6) ( $\beta$ is the kinetic coefficient and $\left.\mu_{\beta}=v \phi /(\beta \Delta)\right)$. In this case the selection condition reads $\mu_{d}=\pi\left(1-\mu_{\beta}\right)^{2} / 2$. We note also that in the case of complete wetting, where the angle $\phi=0$ or extremely small, the evolution of the triple point would be controlled by kinetic effects at the triple junction itself, which are not included in our description.
Eutectoid "dendrite."-Another illustrative example of selection by the triple junction is the eutectoid reaction. We consider here a rather simple model of the eutectoid system, described by the phase diagram in Fig. 4, in order to obtain the equation in the spirit of Eq. (4). We restrict the consideration to the case of an isolated "eutectoid dendrite" as shown in Fig. 2. The concentration fields have jumps on the phase boundaries in eutectoid reactions. As a consequence the difference of concentration $C_{E}-C_{\alpha}$ (source term), and $C_{E}-C_{\beta}=-\left(C_{E}-C_{\alpha}\right)$ (sink term) appear as the amplitudes in front of the integral terms.

Moreover, we also assume that the diffusion constants are equal in all three solid phases. This assumption crucially simplifies the model. The concentration $C_{\gamma}$ far away from the interface is chosen to be $C_{E}$ leading to the symmetry of the shape as presented in Fig. 2. Finally, this system can be described by the integro-differential equation as in the case of classical dendritic growth. Because of the assumed symmetry of the phase diagram and of the shape of the interfaces, we consider only positive values of $x$, i.e., write down the equation only for the $\gamma / \beta$ interface:

$$
\begin{aligned}
\Delta-\frac{d_{0}}{R} \kappa= & -\frac{p}{\pi} \int_{-\infty}^{0} d x^{\prime} e^{-p\left(y-y^{\prime}\right)} K_{0}\left[p \eta\left(x, x^{\prime}\right)\right] \\
& +\frac{p}{\pi} \int_{0}^{\infty} d x^{\prime} e^{-p\left(y-y^{\prime}\right)} K_{0}\left[p \eta\left(x, x^{\prime}\right)\right] .
\end{aligned}
$$

Here $d_{0}$ is the capillary length [10], $\kappa$ is the curvature of the interface which is positive in Fig. 2, and $\Delta=\left(C_{E}-\right.$ $\left.C_{\gamma \beta}^{e}\right) /\left(C_{\beta}-C_{E}\right)$ is the supersaturation. This equation differs from Eq. (4) only by the sign in front of the first integral, which represents the source at the $\alpha / \gamma$ interface,

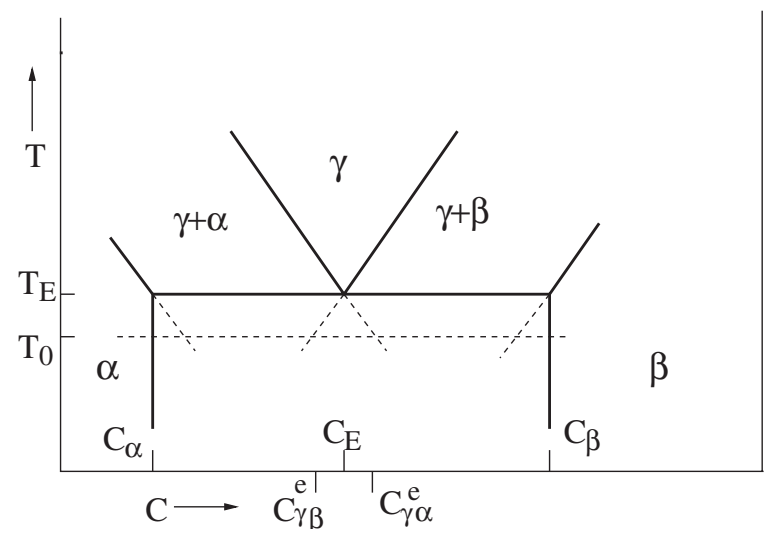

FIG. 4. The schematic phase diagram of the eutectoid reaction. $T_{0}$ is the temperature of the system, $T_{E}$ is the eutectoid temperature. $C_{\alpha}, C_{\beta}$, and $C_{E}$ are the equilibrium concentrations at the eutectoid temperature. For this diagram the eutectoid composition $C_{E}=\left(C_{\alpha}+C_{\beta}\right) / 2$; the lines of the equilibrium of the $\gamma$ and $\alpha$ phases are parallel; the same for the lines of the equilibrium of the $\gamma$ and $\beta$ phases; the equilibrium concentrations at the $\alpha / \beta$ interface, $C_{\alpha}$ and $C_{\beta}$ do not depend on the temperature. 
while the second integral corresponds to the sink at the $\beta / \gamma$ boundary. In other words, this simple modification of the classical equation of dendritic growth already contains the key ingredient of the eutectoid reaction.

In the most relevant case of small Peclet numbers this equation can be further simplified. We stress that the usual relation between the Peclet number and the supersaturation $\Delta \approx \sqrt{\pi p}$, is no longer valid here. In the small Peclet number limit the flux in the $\alpha$ and $\beta$ phases is much larger than in the $\gamma$ phase. Thus, in the asymptotics, one can write $D \Delta / x=-v d x / d y$. This leads to the parabolic asymptotics of the shape, $y=-x^{2} / 2 R$, and to the following relation between the supersaturation and the Peclet number $(p=v R / 2 D), \Delta=2 p$. Thus, for small supersaturation $\Delta$ Eq. (9) reads

$$
2-\sigma \kappa=\frac{1}{2 \pi} \int_{0}^{\infty} d x^{\prime} \ln \left(\frac{\left(x+x^{\prime}\right)^{2}+\left(y-y^{\prime}\right)^{2}}{\left(x-x^{\prime}\right)^{2}+\left(y-y^{\prime}\right)^{2}}\right)
$$

where $\sigma=d_{0} / R p$. Our purpose is to find the shape with parabolic asymptotics and the eigenvalue $\sigma$ for the given angle $\phi$. We solve this nonlinear equation numerically in the spirit of Ref. [9]. The resulting eigenvalue $\sigma=\sigma^{*}(\phi)$ as function of the opening angle is shown in Fig. 5. We notice, that in contrast to the problem of classical dendritic growth, where $\sigma=\sigma^{*}(\phi, p)$ has a singular point at $\phi=$ $\pi / 2$, in the case of the eutectoid reaction, a solution exists even for $\phi=\pi / 2$. Angles $\phi>\pi / 2$ correspond to growth of $\alpha$ and $\beta$ phases from the $\gamma$ phase $\left(T_{0}<T_{E}\right)$, while angles $\phi<\pi / 2$ describe the inverse case $\left(T_{0}>T_{E}\right)$. In the latter case the structure of the interfaces is the same as presented in Fig. 1, with the replacement of $L \rightarrow \gamma$ and $S \rightarrow \alpha, \beta$. The related model of melting of eutectic structures was discussed in [11].

Since the eigenvalue $\sigma^{*}$ is found, the velocity of the process can be written as $v=2 \sigma^{*} D p^{2} / d_{0}$. Consequently, the velocity of eutectoid growth is proportional to $\Delta^{2}$, while for the classical dendritic growth it scales as $\Delta^{4}$.

We are not aware of any experimental results which support the existence of eutectic dendrites. However, we hope that our results may stimulate some new model experiments with transparent materials in order to find this growth mode in eutectic solidification. Specifically, we have in mind free growth experiments (rather than directional solidification) at initial stages using the seed which contains both $\alpha$ and $\beta$ phases. Then, we think, the isolated eutectic dendrite as a growth mode may develop. If the seed contained only one ( $\alpha$ or $\beta$ ) phase then usual dendrite would develop. The growth velocity of such dendrites would be much smaller compared to eutectic dendrites because of the different velocity scalings mentioned above.

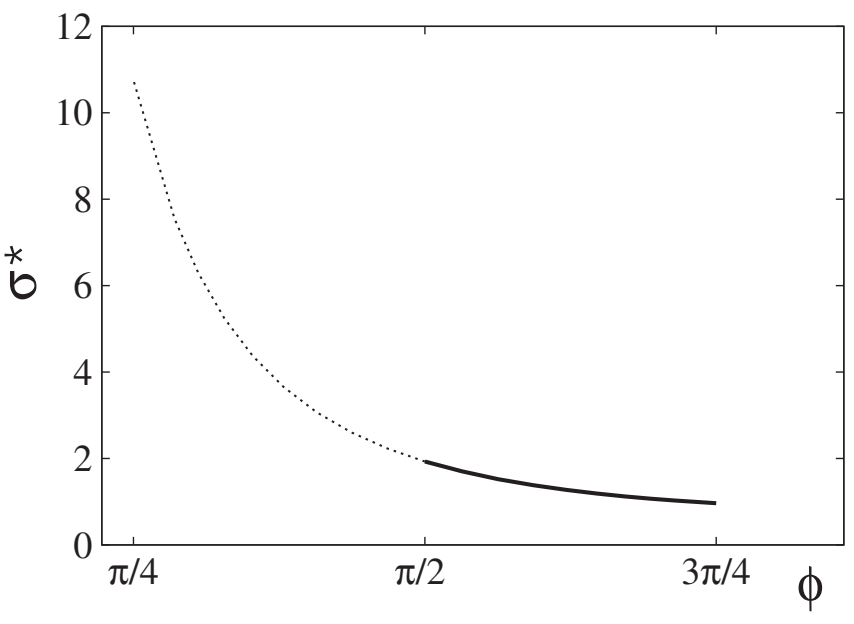

FIG. 5. Eigenvalue $\sigma$ as a function of the opening angle. The solid line $(\phi>\pi / 2)$ corresponds to the growth of the $\alpha$ and $\beta$ phases from the $\gamma$ phase, $T_{0}<T_{E}$. The dotted line $(\phi<\pi / 2)$ to the inverse process, $T_{0}>T_{E}$.

At later stages, the classical lamellar growth should take over.

In conclusion, the selection mechanism due to the presence of the triple junction is very different from the anisotropy effects which are responsible for the selection in the classical theory of dendritic growth. In the regime of small opening angles the problem of velocity selection in melting along the grain boundary has been solved entirely analytically. This exact analytical treatment represents in a very transparent way the structure of the selection theory.

We acknowledge the support by the Deutsche Forschungsgemeinschaft under the Project No. MU 1170/ 4-1.

[1] D. Kessler, J. Koplik, and H. Levine, Adv. Phys. 37, 255 (1988).

[2] E. A. Brener and V.I. Mel'nikov, Adv. Phys. 40, 53 (1991).

[3] M. Ben Amar and E. Brener, Phys. Rev. Lett. 75, 561 (1995).

[4] T. Ihle and H. Müller-Krumbhaar, Phys. Rev. Lett. 70, 3083 (1993).

[5] A. V. Gorbunov, Europhys. Lett. 24, 773 (1993).

[6] D.E. Temkin, Abstracts of ICASP, June 7-10 2005, Stockholm, Sweden (KTH, Stockholm, 2005).

[7] J. S. Langer, Rev. Mod. Phys. 52, 1 (1980).

[8] G. P. Ivantsov, Dokl. Akad. Nauk SSSR 58, 567 (1947).

[9] D. I. Meiron, Phys. Rev. A 33, 2704 (1986).

[10] K. Kassner and C. Misbah, Phys. Rev. A 44, 6513 (1991).

[11] E. A. Brener and D.E. Temkin, Acta Mater. 55, 2785 (2007). 